\title{
Photoreflectance and Raman Study of Surface Electric States on AlGaAs/GaAs Heterostructures
}

\author{
Luis Zamora-Peredo, ${ }^{1}$ Leandro García-González, ${ }^{1}$ Julián Hernández-Torres, ${ }^{1}$ \\ Irving E. Cortes-Mestizo, ${ }^{2}$ Víctor H. Méndez-García, ${ }^{2}$ and Máximo López-López ${ }^{3}$ \\ ${ }^{1}$ Centro de Investigación en Micro y Nanotecnología, Universidad Veracruzana, Calzada Adolfo Ruiz Cortines 455, \\ Fracc. Costa Verde, 94292 Boca del Río, VER, Mexico \\ ${ }^{2}$ Universidad Autónoma de San Luis Potosí, Center for the Innovation and Application of Science and Technology, \\ Sierra Leona 550, Lomas 4a Secc., 78210 San Luis Potosí, SLP, Mexico \\ ${ }^{3}$ Centro de Investigación y Estudios Avanzados del IPN, Apartado Postal 14-740, 07360 Ciudad de México, Mexico
}

Correspondence should be addressed to Luis Zamora-Peredo; luiszamora@uv.mx

Received 1 July 2016; Revised 9 September 2016; Accepted 18 September 2016

Academic Editor: Carlos Andres Palacio

Copyright (C) 2016 Luis Zamora-Peredo et al. This is an open access article distributed under the Creative Commons Attribution License, which permits unrestricted use, distribution, and reproduction in any medium, provided the original work is properly cited.

\begin{abstract}
Photoreflectance (PR) and Raman are two very useful spectroscopy techniques that usually are used to know the surface electronic states in GaAs-based semiconductor devices. However, although they are exceptional tools there are few reports where both techniques were used in these kinds of devices. In this work, the surface electronic states on $\mathrm{AlGaAs} / \mathrm{GaAs}$ heterostructures were studied in order to identify the effect of factors like laser penetration depth, cap layer thickness, and surface passivation over PR and Raman spectra. PR measurements were performed alternately with two lasers ( $532 \mathrm{~nm}$ and $375 \mathrm{~nm}$ wavelength) as the modulation sources in order to identify internal and surface features. The surface electric field calculated by PR analysis decreased whereas the GaAs cap layer thickness increased, in good agreement with a similar behavior observed in Raman measurements $\left(I_{\mathrm{L}-} / I_{\mathrm{LO}}\right.$ ratio). When the heterostructures were treated by Si-flux, these techniques showed contrary behaviors. PR analysis revealed a diminution in the surface electric field due to a passivation process whereas the $I_{\mathrm{L}-} / I_{\mathrm{LO}}$ ratio did not present the same behavior because it was dominated by the depletion layers width (cap layer thickness) and the laser penetration depth.
\end{abstract}

\section{Introduction}

GaAs, with five times higher electron mobility compared to silicon, has a potential to achieve ultrafast electronic and optoelectronic devices. However, this semiconductor suffers from pronounced effects associated with its surface or interfaces: in particular, the large densities of GaAs surface electronic states that pin the Fermi level at midgap and result in large surface recombination velocity $\left(10^{6} \mathrm{~cm} / \mathrm{s}\right)$. In order to eliminate chemical instability that may cause undesired effects, it is well established that the surfaces of GaAs-based devices have to be treated suitably. Frequently, the surfaces of semiconductor devices are passivated in order to stabilize their chemical nature and to eliminate reactivity. Ammonium polysulfide $\left(\mathrm{NH}_{4}\right)_{2} \mathrm{~S}_{x}$ has been frequently used to passivate the surface of GaAs with covalently bonded sulfur atoms
[1-9]. However, sulfur passivation only provides short-term surface stability. In order to get more stability, a silicon interface control layers (Si ICL) approach has been proposed by Hasegawa and Akazawa [10-12] as a complement to high$k$ dielectric oxide surface layers such as $\mathrm{HfO}_{2}$ [13-16], $\mathrm{AlO}_{3}$ $[16,17]$, and $\mathrm{SiO}[18]$. Other studies have been made with selfassembled monolayers (SAMs) of octadecanethiol (ODT) and dodecanethiol (DDT) $[19,20]$.

Specifically, with $\mathrm{AlGaAs} / \mathrm{GaAs}$ heterostructures there are reports about the effect of the surface on the electronic properties. In particular, the relationship between the surface electric field and electron mobility in the two-dimensional electron gas (2DEG) achieved in the AlGaAs/GaAs interface has been studied [21-31]. Other studies with heterojunction bipolar transistors [32], high electron mobility transistors 
[33-35], quantum Hall effect [36], and nanostructure-based devices [37-41] have been reported.

Photoreflectance and Raman spectroscopy are very useful approaches that have been widely used for the study of GaAs-based devices [4, 23-31]. The PR technique has been used to study the $\mathrm{AlGaAs} / \mathrm{GaAs}$ system in numerous reports, which were focused on determining the origin of FranzKeldysh oscillations (FKO) that usually are observed at the PR spectra [24-31]. Today we know that wide-period FKO observed between 1.42 and $1.7 \mathrm{eV}$ are associated with the surface electric field and short-period FKO just above $1.42 \mathrm{eV}$ originate from internal AlGaAs/GaAs interfaces [31]. In the Raman spectroscopy case, there are many reports where it is employed as a useful tool to explore the surface passivation of GaAs films and AlGaAs/GaAs heterostructures. It is well accepted that the relative intensity between longitudinal optical (LO) and coupled plasmon-phonon (L-) modes can reveal useful information about the surface passivation of GaAs [5-7, 20, 42-45].

In this work, we studied a set of $\mathrm{AlGaAs} / \mathrm{GaAs}$ heterostructures by photoreflectance and Raman spectroscopy in order to make a comparative study with both techniques. Samples with different thickness of the GaAs cap layer were grown in order to change the surface electric field. PR measurements with two different laser wavelengths were used in order to identify internal and surfaces features. In addition, we studied the effect generated by the in situ deposition of silicon monolayers (Si ML) over the cap layer of the heterostructure.

\section{Experimental Details}

A set of AlGaAs/GaAs heterostructures was grown on semiinsulating GaAs (100) substrate by molecular beam epitaxy. All samples have a $1 \mu \mathrm{m}$ thick GaAs buffer layer (BL), a first spacer layer (1SL) of $7 \mathrm{~nm}$ undoped $\mathrm{Al}_{x} \mathrm{Ga}_{1-x} \mathrm{As}$, followed by an $80 \mathrm{~nm}$ thick Si-doped AlGaAs barrier (doping with $1.4 \times$ $10^{18}$ atoms $/ \mathrm{cm}^{3}$ ). Next, there is a second spacer layer (2SL) of $7 \mathrm{~nm}$ undoped $\mathrm{Al}_{x} \mathrm{Ga}_{1-x}$ As. Nominal Al concentration of $32 \%$ was used for the AlGaAs layers. Finally, the structure was capped with an undoped GaAs layer (see Figure 1). The thickness of the top layer was 25,60 , and $80 \mathrm{~nm}$ for samples $\mathrm{M} 1, \mathrm{M} 2$, and $\mathrm{M} 3$, respectively. Additionally, two more samples were grown with a $25 \mathrm{~nm}$-GaAs cap layer and an in situ surface passivation treatment with Si-flux in order to get a nominal thickness of 1 and 2 ML, labeled M4 and M5, respectively. When the silicon was deposited the substrate temperature was fixed at $600^{\circ} \mathrm{C}$ and the arsenic flux was maintained in order to get a good quality surface.

Photoreflectance (PR) measurements were carried out alternately with two solid-state lasers as modulation source $(543 \mathrm{~nm}$ and $375 \mathrm{~nm}$ wavelengths, with a maxima output power of 12 and $10 \mathrm{~mW}$, resp.) and chopped with a frequency of $200 \mathrm{~Hz}$. A Sciencetech monochromator of $0.5 \mathrm{~m}$ focal distance was used. The experimental setup used was similar to those that are described elsewhere [23]. Using the $543 \mathrm{~nm}$ laser, it is possible to get a PR signal from the GaAs buffer layer because the penetration depth is close to $120 \mathrm{~nm}$; however with a $375 \mathrm{~nm}$

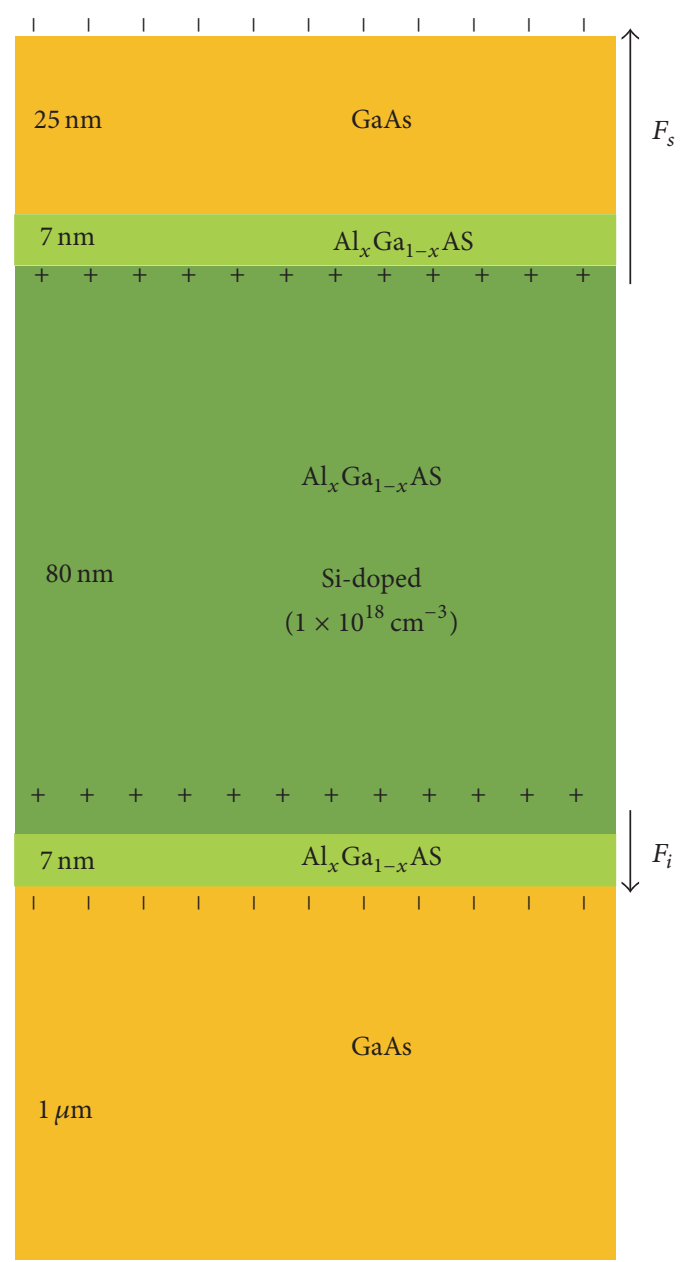

Figure 1: Schematic diagram of semiconductor layers in the heterostructures. $F_{s}$ and $F_{i}$ label the surface and internal electric field region, respectively.

line laser, the penetration depth is reduced significantly $(<50 \mathrm{~nm})$ [46]. All measurements were carried out at room temperature. Raman spectra were acquired with a Thermo Scientific confocal microscopy system arranged in a $180^{\circ}$ backscattering configuration and equipped with a $532 \mathrm{~nm}$ solid-state laser with output power of $10 \mathrm{~mW}, 100 \mathrm{x}$ objective, and a charged-coupled device (CCD). 100 exposures and $10 \mathrm{~s}$ as collection time were used to collect Raman scattering. All spectra were normalized using the LO-GaAs mode intensity.

\section{Results and Discussion}

3.1. Effect of the Capping Layer Thickness. Figure 2 shows room temperature PR spectra of M1 obtained with two different lasers as modulation source: with $532 \mathrm{~nm}$ (black line) and with $375 \mathrm{~nm}$ (red line). In the PR spectrum obtained with $532 \mathrm{~nm}$, it is possible to see three features: a short-period Franz-Keldysh oscillation (s-FKO) at $1.42 \mathrm{eV}$ associated with the GaAs energy band gap, a wide-period Franz-Keldysh oscillation (w-FKO) between 1.42 and $1.6 \mathrm{eV}$, and a wide oscillation associated with the AlGaAs energy band gap. PR 


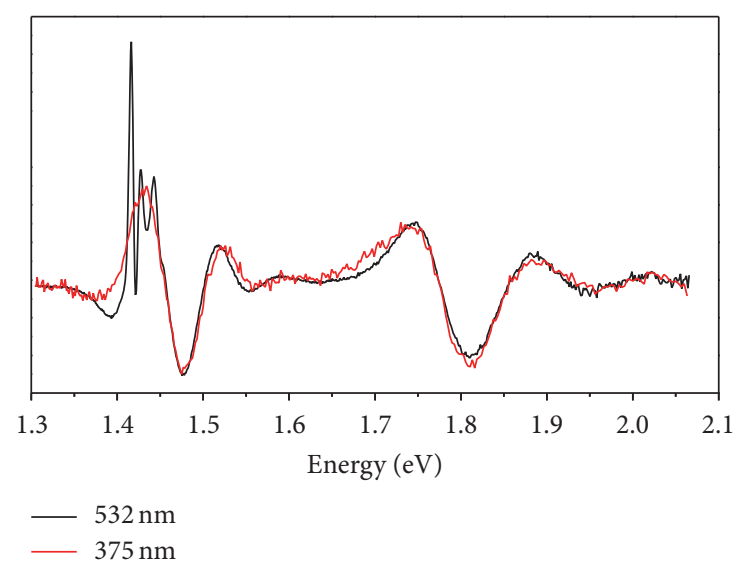

FIGURE 2: PR spectra of M1 heterostructure obtained with $532 \mathrm{~nm}$ (black line) and $375 \mathrm{~nm}$ (red line) lasers as modulation source.

obtained with a $375 \mathrm{~nm}$ laser does not have the first feature, which indicates that it originates from an internal AlGaAs/ GaAs-BL interface considering that the $375 \mathrm{~nm}$ laser has a penetration depth smaller than $50 \mathrm{~nm}$. Bessolov et al. estimated a penetration depth of $108 \mathrm{~nm}$ with a $514.5 \mathrm{~nm}$ line laser and $50.8 \mathrm{~nm}$ for a $457.9 \mathrm{~nm}$ line [6]. The w-FKO is observable with both lasers, which indicates that it originates from the surface GaAs layer. In previous reports this feature has been associated with the surface electric field [31]. Finally, at $1.8 \mathrm{eV}$ we can see the feature originates from the AlGaAs layers, which offers information about energy band gap and consequently of the $\mathrm{Al}$ concentration. Considering that $\mathrm{PR}$ spectra originate mainly from interfaces, it is possible to establish that this oscillation originates from the GaAs/AlGaAs interface nearest to the surface because it is observed with both lasers.

In order to diminish the surface electric field $\left(F_{s}\right)$ originating from the electron migration from the AlGaAs:Si layer to the surface (see Figure 1), the M2 and M3 samples were grown with a GaAs cap layer of 60 and $80 \mathrm{~nm}$ thickness, respectively. Figure 3 shows PR spectra obtained with the $375 \mathrm{~nm}$ laser. The s-FKO feature vanishes for all samples. The w-FKO shows a reduction on its periodicity originating from the diminution of the surface electric field. The feature associated with the AlGaAs layer is only observed in M1 and almost disappears in the M2 and M3 spectra, due to the increase in the distance between the surface and the AlGaAs layer (2SL).

To determine the electric field magnitude associated with the FKO, we considered the asymptotic modulation expression for the electroreflectance proposed by Aspnes and Studna [47]:

$$
\begin{gathered}
\frac{\Delta R}{R} \propto \frac{1}{E^{2}\left(E-E_{g}\right)} \exp \left[\frac{-2 \Gamma \sqrt{E-E_{g}}}{(\hbar \theta)^{3 / 2}}\right] \\
\cdot \cos \left[\frac{4}{3}\left(\frac{E-E_{g}}{\hbar \theta}\right)^{3 / 2}+\chi\right],
\end{gathered}
$$
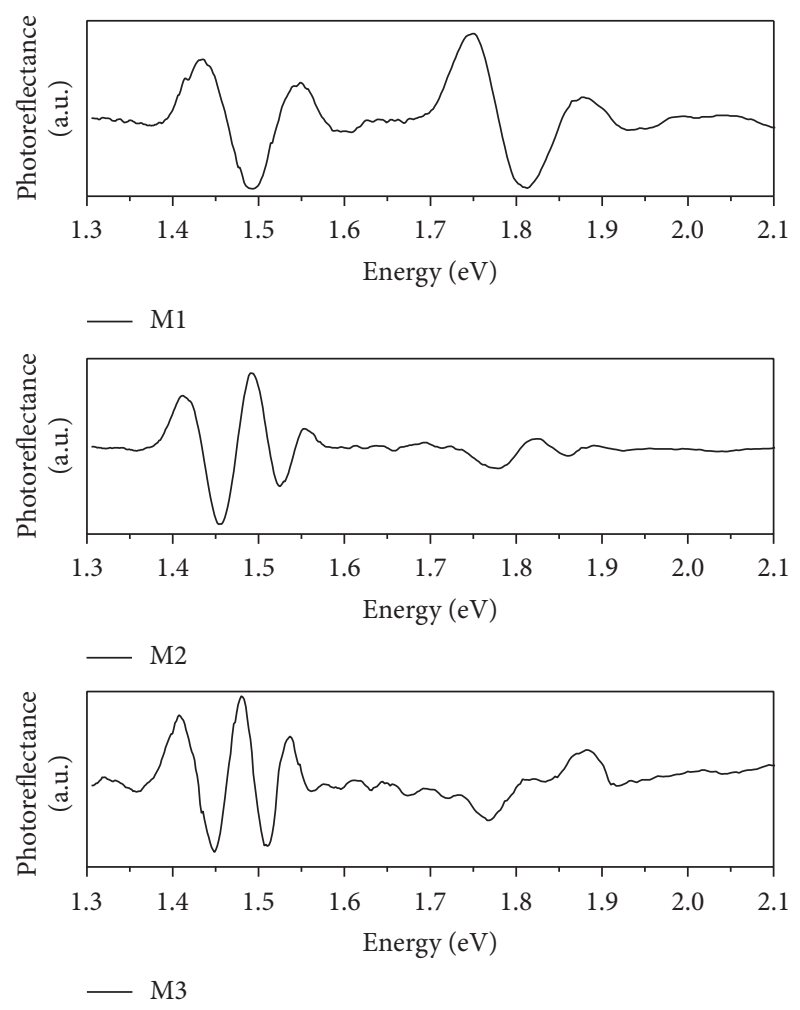

FIgURE 3: PR spectra of M1, M2, and M3 heterostructures, capped with 25,60 , and $80 \mathrm{~nm}$ GaAs layer, respectively. The $375 \mathrm{~nm}$ laser was used as modulation source.

where $\hbar \theta$ is the electrooptic energy, $\Gamma$ is the linewidth, $E_{g}$ is the band gap energy, and $\chi$ is an arbitrary phase factor. The electric field $F$ is related to $\hbar \theta$ by the expression

$$
\hbar \theta=\left(\frac{e^{2} \hbar^{2} F^{2}}{2 \mu}\right)^{1 / 3},
$$

where $\mu$ is the electron-hole reduced mass and $e$ is the electron charge.

In this model, the position of an $n$th extreme in the FKO is given by

$$
n \pi=\frac{4}{3}\left(\frac{E_{n}-E_{g}}{\hbar \theta}\right)^{3 / 2}+\chi
$$

where $n$ is the index and $E_{n}$ is the corresponding energy.

Equation (3) can be rearranged as

$$
\frac{4}{3 \pi}\left(E-E_{g}\right)^{3 / 2}=(\hbar \theta)^{3 / 2} n-\frac{(\hbar \theta) \chi}{\pi} .
$$

As we can see, (4) corresponds to a linear function with slope $(\hbar \theta)^{3 / 2}$, which can be determined using experimental data by a linear fitting of the plot of $(4 / 3 \pi)\left(E-E_{g}\right)^{3 / 2}$ versus the index number $n$. Next, $F$ can be determined using (2).

Figure 4 shows the linear fit obtained with experimental data from the w-FKO extreme in PR spectra of M1, M2, and M3 where it is evidence of the reduction of the surface electric 
TABLE 1: Cap layer thickness, surface electric field $\left(F_{s}\right)$ obtained from PR measurements, and $I_{\mathrm{L}-} / I_{\mathrm{LO}}$ ratio calculated from Raman measurements.

\begin{tabular}{lcccc}
\hline Sample & Cap $(\mathrm{nm})$ & $\mathrm{Si}(\mathrm{ML})$ & $F_{s}\left(10^{7} \mathrm{~V} / \mathrm{m}\right)$ & $I_{\mathrm{L}-} / I_{\mathrm{LO}}$ \\
\hline M1 & 25 & 0 & 5.99 & 0.1699 \\
M2 & 60 & 0 & 3.57 & 0.1407 \\
M3 & 80 & 0 & 3.40 & 0.1345 \\
M4 & 25 & 1 & 5.91 & 0.1896 \\
M5 & 25 & 2 & 5.08 & 0.1772 \\
\hline
\end{tabular}

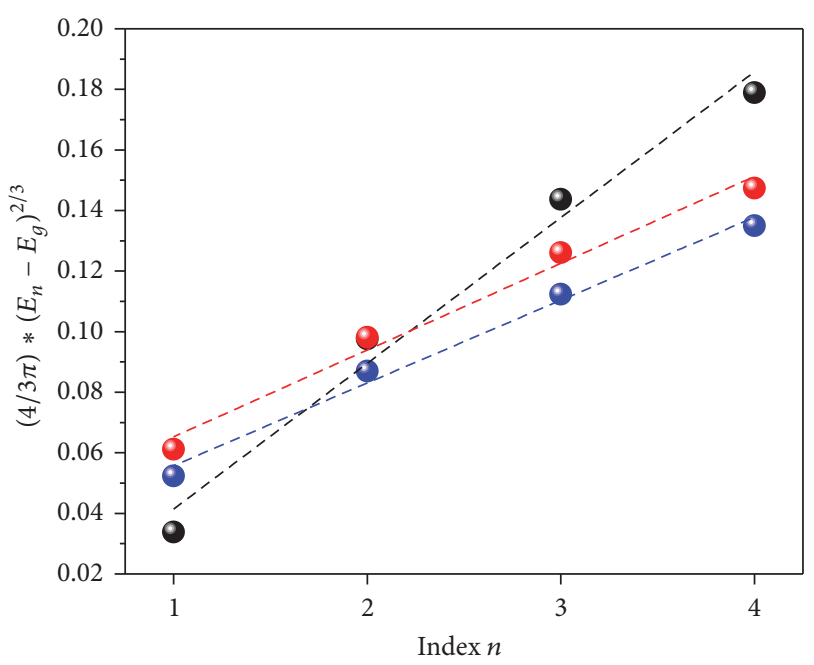

$\begin{array}{ll}\text { - } & M 1 \\ \text { - } & \text { M2 } \\ \text { - } & \text { M3 }\end{array}$

FIGURE 4: Linear fit in w-FKO analysis from PR spectra of M1, M2, and $\mathrm{M} 3$.

field $\left(F_{s}\right)$ as the cap layer thickness increases. As we can see in Table 1, the magnitude of $F_{s}$ decreases from 5.99 to 3.57 and $3.40 \times 10^{5} \mathrm{~V} / \mathrm{cm}$ (reduction of $43.2 \%$ ) for M1, M2, and M3, respectively.

Figure 5 shows Raman spectra of heterostructures M1, M2, and M3 (cap layer thickness of 25, 60, and $80 \mathrm{~nm}$, resp.) where there are observed four vibration modes: the coupled plasmon-phonon (L-) and longitudinal optical (LO) from the GaAs cap layer localized at 268 and $291 \mathrm{~cm}^{-1}$, respectively and two modes originating from the AlGaAs layers (LO GaAs-like and LO AlAs-like) located at 281 and $377 \mathrm{~cm}^{-1}$, respectively. All Raman spectra were normalized at LO GaAs mode intensity. L- mode intensity is lower than the LO GaAs mode because the GaAs layers (cap and $\mathrm{BL}$ ) are undoped. The effect of increasing the cap layer thickness is palpable with the LO GaAs-like and LO AlAs-like modes intensity because both decrease as the surface GaAs thickness increases.

In $\mathrm{n}$-doped GaAs films, the LO peak $\left(291 \mathrm{~cm}^{-1}\right)$ is attributed to the surface depletion layer whereas L- mode originates from the bulk where free carriers exist [3-7, 20, 42-44]. In this case, the cap layer thickness is the same as the wide depletion layer as we can see in Figure 1. Then the LO intensity $\left(I_{\mathrm{LO}}\right)$ will be increased as the cap layer thickness increases and

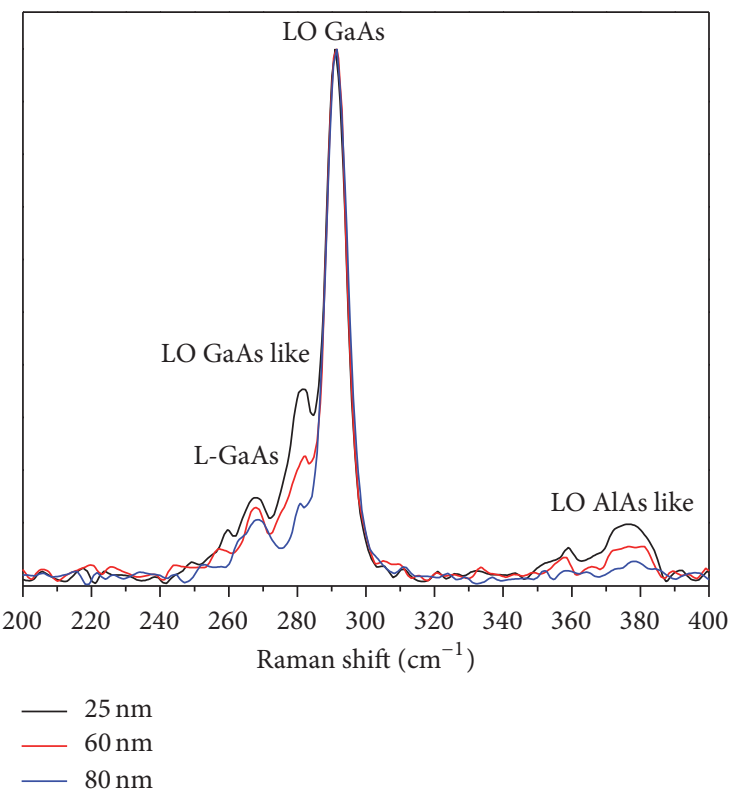

Figure 5: Raman scattering of M1, M2, and M3 heterostructures, capped with GaAs layer of 25, 60, and $80 \mathrm{~nm}$, respectively.

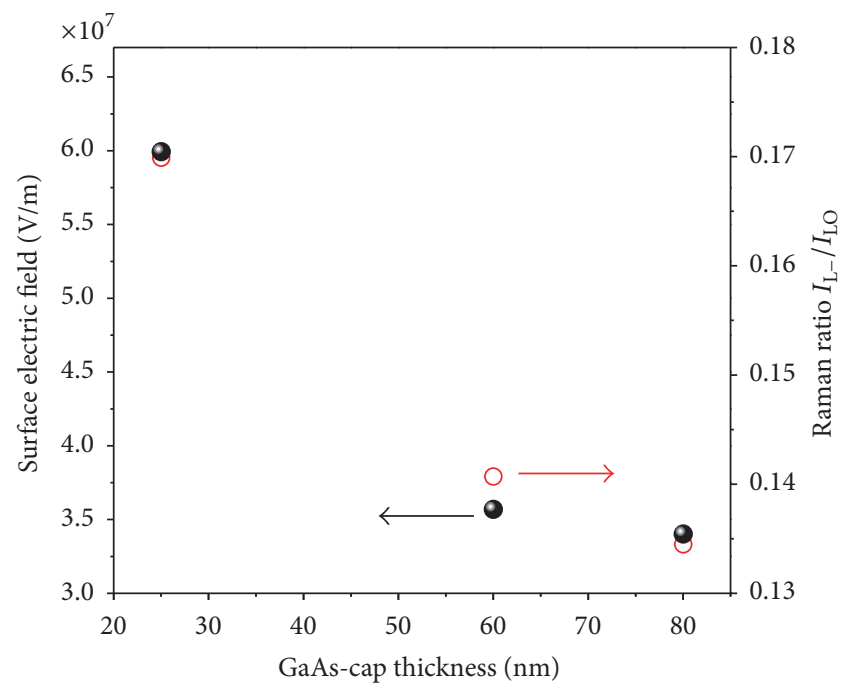

FIGURE 6: Behavior comparison of the surface electric field obtained by PR (black point) and Raman ratio $I_{\mathrm{L}-} / I_{\mathrm{LO}}$ (red circle) as a function of cap layer thickness.

therefore we can use the $I_{\mathrm{L}-} / I_{\mathrm{LO}}$ ratio to study the surface states. A summary of the measured values for the $I_{\mathrm{L}-} / I_{\mathrm{LO}}$ ratio acquired from Raman spectra in Figure 5 is presented in Table 1. Figure 6 plots the surface electric field obtained by PR (black point) and Raman ratio $I_{\mathrm{L}-} / I_{\mathrm{LO}}$ (red circle) as a function of cap layer thickness. As we can see, both techniques show a similar behavior of the surface electric field in these heterostructures. Similar comportment of the surface electric field has been found by Kudrawiec et al. in GaN Van Hoof structures studied by contactless electroreflectance [48].

3.2. Effect of Si Passivation. An analogous study was made with heterostructures passivated with $\mathrm{Si}-\mathrm{ML}$. In this case, 

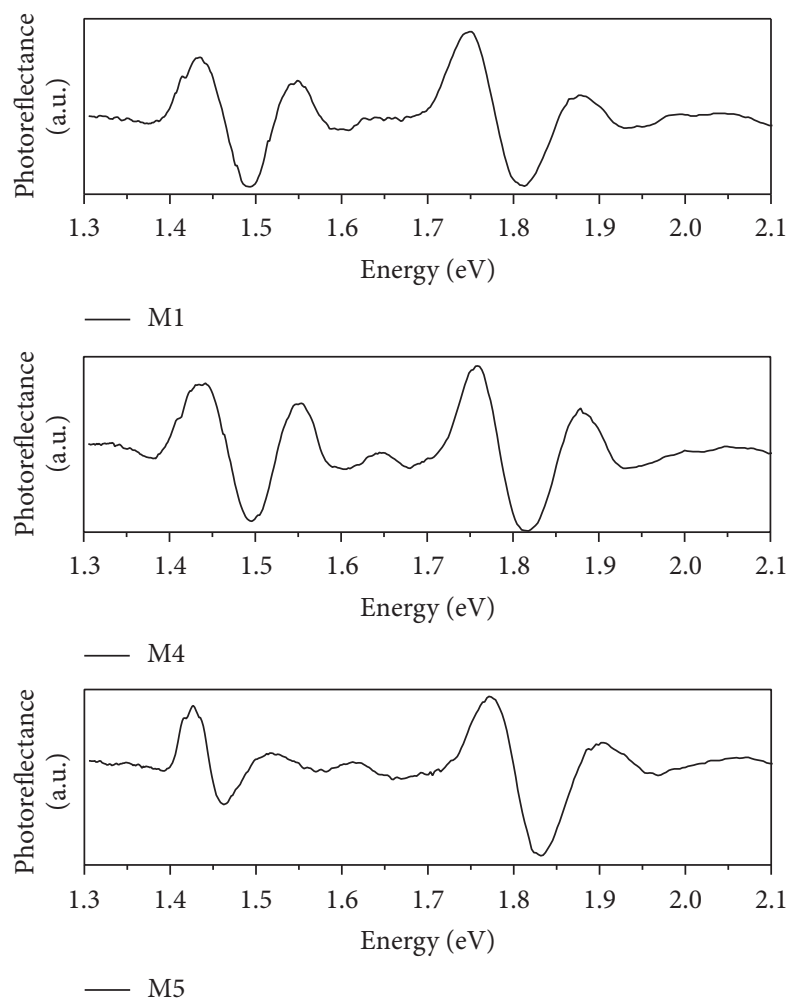

FIGURE 7: PR spectra of M1, M4, and M5 heterostructures passivated with 0,1 , and 2 silicon monolayers, respectively. The $375 \mathrm{~nm}$ laser was used as modulation source.

the cap layer thickness remains constant and therefore a reduction of the surface states density is expected due to the passivation process originating from the formation of a $\mathrm{SiO}$ monolayer when the samples were exposed to the atmosphere. Figure 7 shows PR spectra of M1, M4, and M5 in order to compare samples without (M1) and with Si-ML (M4 and M5). The oscillation at $E>1.7 \mathrm{eV}$ associated with the AlGaAs layer did not disappear because the cap layer thickness was $25 \mathrm{~nm}$ for the three samples and the laser penetration depth is bigger. The w-FKO period has a similar behavior for M1 and M4; however it presents a notable change in M5. Linear fit with experimental data from w-FKO extreme is shown in Figure 8. As we can see in Table 1, the surface electric field magnitude changes from 5.99 to 5.91 and 5.08 $\times 10^{5} \mathrm{~V} / \mathrm{cm}$ for M1, M4, and M5, respectively. These results suggest that the surface passivation process is occurring but could be insufficient to eliminate the surface state because the $\mathrm{Si}$ deposition was made at high temperature $\left(\sim 600^{\circ} \mathrm{C}\right)$.

Figure 9 shows Raman spectra of heterostructures $\mathrm{Ml}$, M4, and M5 (treated with 0,1 , and 2 Si-ML, resp.) where the same four vibration modes that were observed in Figure 5 are observed. All Raman spectra were normalized at LO GaAs mode. When the heterostructure is treated with $\mathrm{Si}$ monolayers it is possible to see some changes. In this case the LO AlAs-like peak originating from the AlGaAs layer does not decrease because the cap layer is of a thicknesses of $25 \mathrm{~nm}$ for the three samples. The $I_{\mathrm{L}-} / I_{\mathrm{LO}}$ ratio changes from 0.170 to 0.189 and 0.177 for M1, M4, and M5, respectively (Table 1).

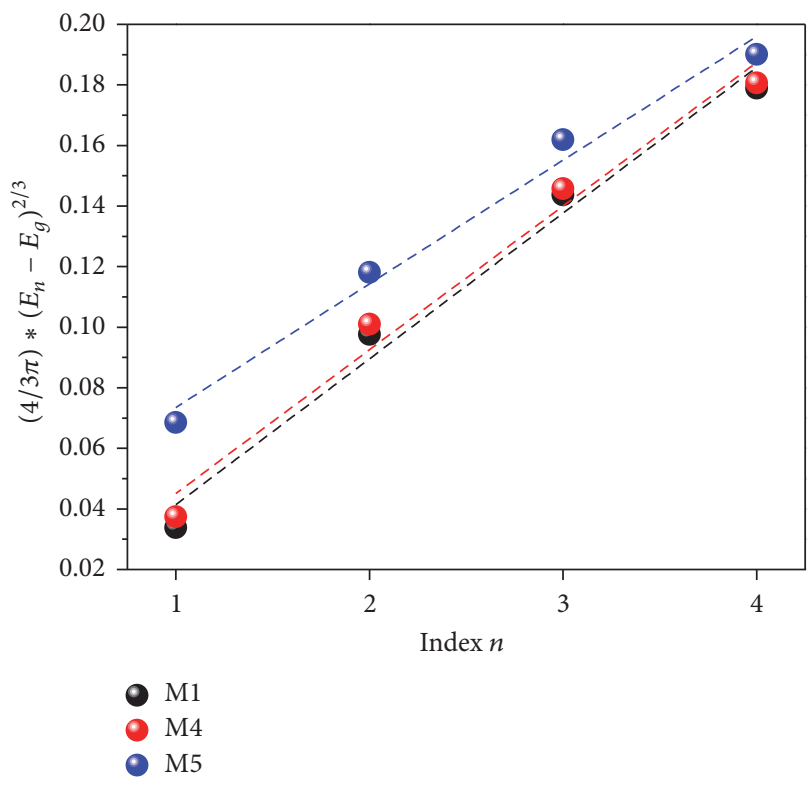

FIGURE 8: Linear fit in w-FKO analysis from PR spectra of M1, M4, and M5.

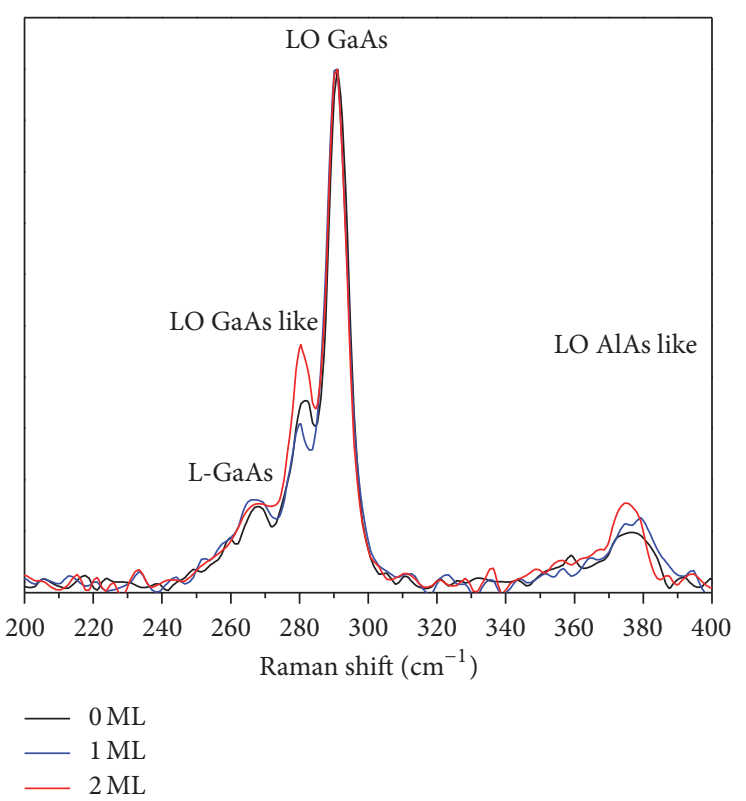

FIGURE 9: Raman scattering of M1, M4, and M5 heterostructures capped with a $25 \mathrm{~nm}$ GaAs layer and passivated with 0,1 , and $2 \mathrm{Si}$ $\mathrm{ML}$, respectively. A $532 \mathrm{~nm}$ laser was used as excitation source.

Figure 10 plots the surface electric field obtained by PR (black point) and Raman intensity ratio $I_{\mathrm{L}-} / I_{\mathrm{LO}}$ (red circle) as a function of the $\mathrm{Si}$ monolayers. In this case, the PR measurement analysis gives a slight decrease of the surface electric field for samples with 1 and 2 Si-ML but Raman scattering insinuates a contrary situation. Considering that the surface depletion layer remains constant, we would expect an unchanged ratio $I_{\mathrm{L}-} / I_{\mathrm{LO}}$. This disagreement between PR and Raman spectroscopy could be associated with the laser penetration depth because the laser used in Raman system is 


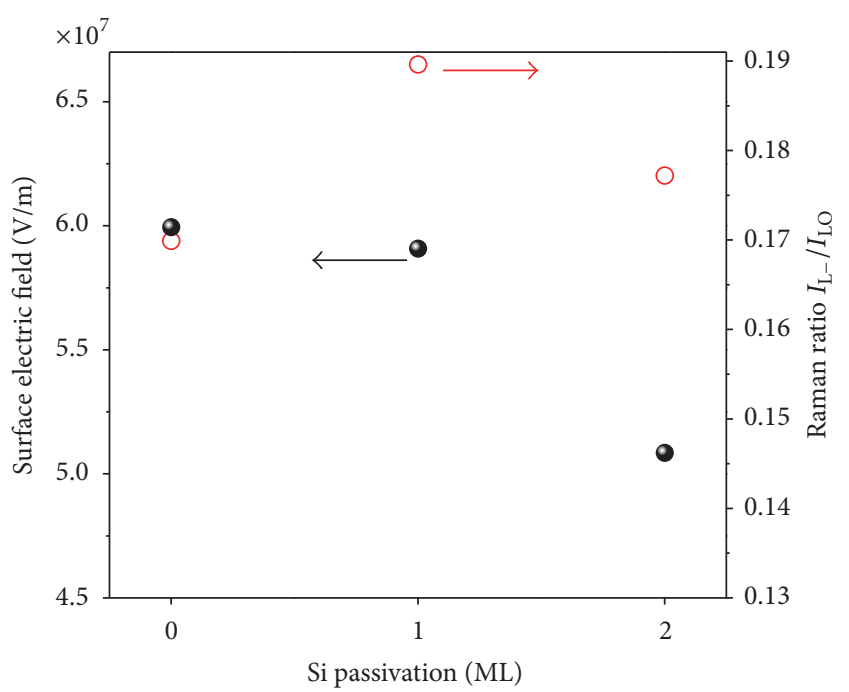

FIGURE 10: Behavior comparison of the surface electric field obtained by PR (black point) and Raman ratio $I_{\mathrm{L}^{-}} / I_{\mathrm{LO}}$ (red circle) as a function of Si-ML.

similar to PR measurements (Figure 2) so that it excites the GaAs buffer layer. Consequently, both cap and deeper GaAs layers influence Raman spectra. In previous studies with these kinds of heterostructures, we found that if the surface electric field decreases (as is suggested by PR measurements) a wider depletion zone is originated in the $\mathrm{AlGaAs} / \mathrm{GaAs}$ BL interface [49], which changes the Raman intensity ratio $I_{\mathrm{L}-} / I_{\mathrm{LO}}$. That means that PR measurements are the best approach to study the surface electric field because Raman spectra are influenced by deeper GaAs interface.

The PR analysis in this work is in good agreement with the engineering of electric field distribution in $\mathrm{AlGaN} / \mathrm{GaN}$ heterostructures that widely has been studied by other authors where electroreflectance spectroscopy has shown similar behavior when the cap layer is modified $[48,50,51]$. The above suggests that PR spectroscopy is an excellent tool that could be used to explore more complex heterostructures like GaN/graphene/Si [52] or GaAs/Graphene/Si [53] where the internal electric field can give information about crystal quality.

\section{Conclusions}

The surface electronic states on $\mathrm{AlGaAs} / \mathrm{GaAs}$ heterostructures were studied by photoreflectance and Raman spectroscopy techniques. The surface electric field calculated by PR analysis decreased whereas the GaAs cap layer thickness increases in good agreement with a similar behavior observed in Raman measurements $\left(I_{\mathrm{L}-} / I_{\mathrm{LO}}\right.$ ratio). When the heterostructures were treated with a Si-flux, these techniques showed contrary behaviors originating from the penetration depth of the laser used in the PR and Raman measurements. PR analysis found a slow diminishment in the surface electric field whereas the $I_{\mathrm{L}-} / I_{\mathrm{LO}}$ ratio observed in Raman analysis showed higher values than the sample without a Si-ML. This work illustrates how it is possible to use PR and Raman spectroscopy to study the surface electronic states in AlGaAs/ GaAs heterostructures and the passivation process in this kind of semiconductor device.

\section{Competing Interests}

The authors declare that they have no competing interests.

\section{Acknowledgments}

The authors want to thank DGDAIE-UV, DGI-UV, and SENER-CONACYT for their support of this work.

\section{References}

[1] Y. Nannichi, J.-F. Fan, H. Oigawa, and A. Koma, "A model to explain the effective passivation of the GaAs surface by $\left(\mathrm{NH}_{4}\right)_{2} \mathrm{~S}_{x}$ treatment," Japanese Journal of Applied Physics, vol. 27, no. 12, pp. L2367-L2369, 1988.

[2] H. Oigawa, J.-F. Fan, Y. Nannichi, H. Sugahara, and M. Oshima, "Universal passivation effect of $\left(\mathrm{NH}_{4}\right)_{2} \mathrm{~S}_{x}$ treatment on the surface of III-V compound semiconductors," Japanese Journal of Applied Physics, vol. 30, no. 3, pp. L322-L325, 1991.

[3] R. V. Ghita, C. C. Negrila, C. Cotirlan, and C. Logofatu, "On the passivation of GaAs surface by sulfide compounds," Digest Journal of Nanomaterials and Biostructures, vol. 8, no. 3, pp. 1335-1344, 2013.

[4] P. Jin, S. H. Pan, Y. G. Li, C. Z. Zhang, and Z. G. Wang, "Electronic properties of sulfur passivated undoped- $\mathrm{n}^{+}$type GaAs surface studied by photoreflectance," Applied Surface Science, vol. 218, no. 1-4, pp. 210-214, 2003.

[5] X. Chen, X. Si, and V. Malhotra, "Measurement of reduced surface barrier height in sulfur passivated InP and GaAs using Raman spectroscopy," Journal of the Electrochemical Society, vol. 140, no. 7, pp. 2085-2088, 1993.

[6] V. N. Bessolov, M. V. Lebedev, and D. R. T. Zahn, "Raman scattering study of surface barriers in GaAs passivated in alcoholic sulfide solutions," Journal of Applied Physics, vol. 82, no. 5, pp. 2640-2642, 1997.

[7] T. Błachowicz, G. Salvan, D. R. T. Zahn, and J. Szuber, "MicroRaman spectroscopy of disordered and ordered sulfur phases on a passivated GaAs surface," Applied Surface Science, vol. 252, no. 21, pp. 7642-7646, 2006.

[8] M. V. Lebedev, "Passivation at semiconductor/electrolyte interface: role of adsorbate solvation and reactivity in surface atomic and electronic structure modification of III-V semiconductor," Applied Surface Science, vol. 254, no. 24, pp. 8016-8022, 2008.

[9] J. S. Ha, S.-J. Park, S.-B. Kim, and E.-H. Lee, "Correlation of surface morphology with chemical structures of sulfur-passivated GaAs(100) investigated by scanning tunneling microscopy and $\mathrm{x}$-ray photoelectron spectroscopy," Journal of Vacuum Science and Technology A: Vacuum, Surfaces and Films, vol. 13, no. 3, pp. 646-651, 1995.

[10] H. Hasegawa, "MBE growth and applications of silicon interface control layers," Thin Solid Films, vol. 367, no. 1-2, pp. 58-67, 2000.

[11] M. Akazawa and H. Hasegawa, "Formation of ultrathin $\mathrm{SiN}_{x} / \mathrm{Si}$ interface control double layer on (001) and (111) GaAs surfaces for ex situ deposition of high-k dielectrics," Journal of Vacuum Science and Technology B: Microelectronics and Nanometer Structures, vol. 25, no. 4, pp. 1481-1490, 2007. 
[12] H. Hasegawa and M. Akazawa, "Surface passivation technology for III-V semiconductor nanoelectronics," Applied Surface Science, vol. 255, no. 3, pp. 628-632, 2008.

[13] D. J. Webb, J. Fompeyrine, S. Nakagawa et al., "In-situ MBE Si as passivating interlayer on $\mathrm{GaAs}$ for $\mathrm{HfO}_{2}$ MOSCAP's: effect of GaAs surface reconstruction," Microelectronic Engineering, vol. 84, no. 9-10, pp. 2142-2145, 2007.

[14] C. Marchiori, D. J. Webb, C. Rossel et al., "H plasma cleaning and a-Si passivation of GaAs for surface channel device applications," Journal of Applied Physics, vol. 106, no. 11, Article ID 114112, 2009.

[15] M. El Kazzi, D. J. Webb, L. Czornomaz et al., "1.2 nm capacitance equivalent thickness gate stacks on Si-passivated GaAs," Microelectronic Engineering, vol. 88, no. 7, pp. 1066-1069, 2011.

[16] Y. Xuan, H.-C. Lin, and P. D. Ye, "Simplified surface preparation for GaAs passivation using atomic layer-deposited high- $\kappa$ dielectrics," IEEE Transactions on Electron Devices, vol. 54, no. 8, pp. 1811-1817, 2007.

[17] C. L. Hinkle, A. M. Sonnet, E. M. Vogel et al., "Frequency dispersion reduction and bond conversion on $\mathrm{n}$-type GaAs by in situ surface oxide removal and passivation," Applied Physics Letters, vol. 91, no. 16, Article ID 163512, 2007.

[18] D. L. Winn, M. J. Hale, T. J. Grassman, A. C. Kummel, R. Droopad, and M. Passlack, "Direct and indirect causes of Fermi level pinning at the $\mathrm{SiO} / \mathrm{GaAs}$ interface," Journal of Chemical Physics, vol. 126, no. 8, Article ID 084703, 2007.

[19] H. A. Budz, M. C. Biesinger, and R. R. LaPierre, "Passivation of GaAs by octadecanethiol self-assembled monolayers deposited from liquid and vapor phases," Journal of Vacuum Science and Technology B: Microelectronics and Nanometer Structures, vol. 27, no. 2, pp. 637-648, 2009.

[20] C. L. Mcguiness, A. Shaporenko, M. Zharnikov, A. V. Walker, and D. L. Allara, "Molecular self-assembly at bare semiconductor surfaces: Investigation of the chemical and electronic properties of the alkanethiolate-GaAs(001) interface," Journal of Physical Chemistry C, vol. 111, no. 11, pp. 4226-4234, 2007.

[21] F. Castro, B. Nabet, and X. Chen, "Closed-form electric-field profile model for AlGaAs/GaAs heterostructures," Journal of Applied Physics, vol. 92, no. 1, pp. 218-222, 2002.

[22] R. A. Khabibullin, I. S. Vasil'evskii, G. B. Galiev et al., "Effect of the built-in electric field on optical and electrical properties of AlGaAs/InGaAs/GaAs P-HEMT nanoheterostructures," Semiconductors, vol. 45, no. 5, pp. 657-662, 2011.

[23] J. Misiewicz, P. Sitarek, G. Sęk, and R. Kudrawiec, "Semiconductor heterostructures and device structures investigated by photoreflectance spectroscopy," Materials Science, vol. 21, no. 3, pp. 263-320, 2003.

[24] N. Pan, X. L. Zheng, H. Hendriks, and J. Carter, "Photoreflectance characterization of AlGaAs/GaAs modulation-doped heterostructures," Journal of Applied Physics, vol. 68, no. 5, pp. 2355-2360, 1990.

[25] R. A. Novellino, C. Vazquez-López, A. A. Bernussi et al., "On the origin of Franz-Keldysh oscillations in AlGaAs/GaAs modulation-doped heterojunctions," Journal of Applied Physics, vol. 70, no. 10, pp. 5577-5581, 1991.

[26] J. A. N. T. Soares, D. Beliaev, R. Enderlein, L. M. R. Scolfaro, M. Saito, and J. R. Leite, "Photoreflectance investigations of semiconductor device structures," Materials Science and Engineering $B$, vol. 35, no. 1-3, pp. 267-272, 1995.
[27] I. Hwang, J.-E. Kim, H. Y. Park, and S. K. Noh, "Photoreflectance study of etching and annealing effect on AlGaAsGaAs heterostructure," Solid State Communications, vol. 103, no. 1, pp. 13, 1997.

[28] J. A. N. T. Soares, R. Enderlein, D. Beliaev, J. R. Leite, and M. Saito, "Photoreflectance spectra from GaAs HEMT structures reinvestigated: solution of an old controversy," Semiconductor Science and Technology, vol. 13, no. 12, pp. 1418-1425, 1998.

[29] E. Estacio, M. Bailon, A. Somintac, R. Sarmiento, and A. Salvador, "Observation of high junction electric fields in modulation-doped GaAs/AlGaAs heterostructures by room temperature photoreflectance spectroscopy," Journal of Applied Physics, vol. 91, no. 6, pp. 3717-3720, 2002.

[30] H. Takeuchi, Y. Kamo, Y. Yamamoto, T. Oku, M. Totsuka, and M. Nakayama, "Photovoltaic effects on Franz-Keldysh oscillations in photoreflectance spectra: application to determination of surface Fermi level and surface recombination velocity in undoped GaAs/n-type GaAs epitaxial layer structures," Journal of Applied Physics, vol. 97, no. 6, Article ID 063708, 2005.

[31] L. Zamora-Peredo, I. E. Cortes-Mestizo, L. García-González et al., "Determination of surface electric potential by photoreflectance spectroscopy of HEMT heterostructures," Journal of Crystal Growth, vol. 378, pp. 100-104, 2013.

[32] R. T. Yoshioka, L. E. M. de Barros Jr., J. A. Diniz, and J. W. Swart, "Improving performance of microwave AlGaAs/GaAs HBTs using novel SiNx passivation process," in Proceedings of the SBMO \& IEEE MTT-S, APS and LEOS-International Microwave and Optoelectronics Conference (IMOC '99), vol. 1, pp. 108-111, Rio de Janeiro, Brazil, August 1999.

[33] J.-H. Oh, W.-S. Sul, H.-J. Han et al., "Effects of silicon-nitride passivation on the electrical behavior of $0.1-\mu \mathrm{m}$ pseudomorphic high-electron-mobility transistors," Journal of the Korean Physical Society, vol. 44, no. 4, pp. 899-903, 2004.

[34] D. J. Carrad, A. M. Burke, P. J. Reece et al., "The effect of $\left(\mathrm{NH}_{4}\right)_{2} \mathrm{~S}_{x}$ passivation on the (311)A GaAs surface and its use in $\mathrm{AlGaAs} / \mathrm{GaAs}$ heterostructure devices," Journal of Physics: Condensed Matter, vol. 25, no. 32, Article ID 325304, 2013.

[35] H.-C. Chiu, Y.-C. Huang, C.-W. Chen, and L.-B. Chang, "Electrical characteristics of passivated Pseudomorphic HEMTs with $\mathrm{P}_{2} \mathrm{~S}_{5} /\left(\mathrm{NH}_{4}\right)_{2} \mathrm{~S}_{\mathrm{X}}$ pretreatment," IEEE Transactions on Electron Devices, vol. 55, no. 3, pp. 721-726, 2008.

[36] G. Kopnov, V. Y. Umansky, H. Cohen, D. Shahar, and R. Naaman, "Effect of the surface on the electronic properties of a two-dimensional electron gas as measured by the quantum Hall effect," Physical Review B, vol. 81, no. 4, Article ID 045316, 2010.

[37] C.-C. Chang, C.-Y. Chi, M. Yao et al., "Electrical and optical characterization of surface passivation in GaAs nanowires," Nano Letters, vol. 12, no. 9, pp. 4484-4489, 2012.

[38] H. J. Joyce, P. Parkinson, N. Jiang et al., "Electron mobilities approaching bulk limits in 'surface-free' GaAs nanowires," Nano Letters, vol. 14, no. 10, pp. 5989-5994, 2014.

[39] P. A. Alekseev, M. S. Dunaevskiy, V. P. Ulin et al., "Nitride surface passivation of GaAs nanowires: impact on surface state density," Nano Letters, vol. 15, no. 1, pp. 63-68, 2015.

[40] S. Mokkapati, D. Saxena, N. Jiang, L. Li, H. H. Tan, and C. Jagadish, "An order of magnitude increase in the quantum efficiency of (Al)GaAs nanowires using hybrid photonic-plasmonic modes," Nano Letters, vol. 15, no. 1, pp. 307-312, 2015.

[41] J. L. Boland, S. Conesa-Boj, P. Parkinson et al., "Modulation doping of GaAs/AlGaAs core-shell nanowires with effective 
defect passivation and high electron mobility," Nano Letters, vol. 15, no. 2, pp. 1336-1342, 2015.

[42] L. A. Farrow, C. J. Sandroff, and M. C. Tamargo, "Raman scattering measurements of decreased barrier heights in GaAs following surface chemical passivation," Applied Physics Letters, vol. 51, no. 23, pp. 1931-1933, 1987.

[43] A. Osherov, M. Matmor, N. Froumin, N. Ashkenasy, and Y. Golan, "Surface termination control in chemically deposited PbS films: nucleation and growth on GaAs(111)A and GaAs(111)B," Journal of Physical Chemistry C, vol. 115, no. 33, pp. 16501-16508, 2011.

[44] S. L. Peczonczyk, J. Mukherjee, A. I. Carim, and S. Maldonado, "Wet chemical functionalization of III-V semiconductor surfaces: alkylation of gallium arsenide and gallium nitride by a Grignard reaction sequence," Langmuir, vol. 28, no. 10, pp. 4672-4682, 2012.

[45] S. Arab, C.-Y. Chi, T. Shi et al., "Effects of surface passivation on twin-free GaAs nanosheets," ACS Nano, vol. 9, no. 2, pp. 13361340, 2015.

[46] L. Pavesi and M. Guzzi, "Photoluminescence of $\mathrm{Al}_{x} \mathrm{Ga}_{1-x} \mathrm{As}$ alloys," Journal of Applied Physics, vol. 75, no. 10, pp. 4779-4842, 1994.

[47] D. E. Aspnes and A. A. Studna, "Schottky-barrier electroreflectance: application to GaAs," Physical Review B, vol. 7, no. 10, pp. 4605-4625, 1973.

[48] R. Kudrawiec, M. Gladysiewicz, L. Janicki et al., "Contactless electroreflectance studies of Fermi level position on c-plane GaN surface grown by molecular beam epitaxy and metalorganic vapor phase epitaxy," Applied Physics Letters, vol. 100, no. 18, Article ID 181603, 2012.

[49] L. Zamora-Peredo, I. Cortes-Mestizo, L. García-Gonzáez et al., "Optical and electrical study of cap layer effect in QHE devices with double-2DEG," MRS Proceedings, vol. 1617, pp. 31-36, 2013.

[50] E. Janicki, R. Kudrawiec, K. Pakula, R. Stępniewski, and J. Misiewicz, "Contactless electroreflectance studies of surface potential barrier in $\mathrm{AlGaN} / \mathrm{n}-\mathrm{AlGaN}$ structures with various $\mathrm{Al}$ concentrations," Physica Status Solidi B, vol. 252, no. 5, pp. 10381042, 2015.

[51] M. Gladysiewicz, L. Janicki, J. Misiewicz et al., "Engineering of electric field distribution in GaN(cap)/AlGaN/GaN heterostructures: theoretical and experimental studies," Journal of Physics D: Applied Physics, vol. 49, no. 34, Article ID 345106, 2016.

[52] Y. Alaskar, S. Arafin, D. Wickramaratne et al., "Towards van der waals epitaxial growth of GaAs on Si using a graphene buffer layer," Advanced Functional Materials, vol. 24, no. 42, pp. 66296638, 2014.

[53] J. Kim, C. Bayram, H. Park et al., "Principle of direct van der Waals epitaxy of single-crystalline films on epitaxial graphene," Nature Communications, vol. 5, article 4836, 2014. 

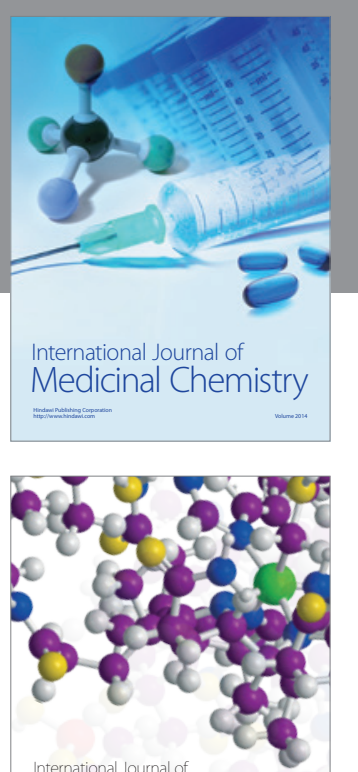

Carbohydrate Chemistry

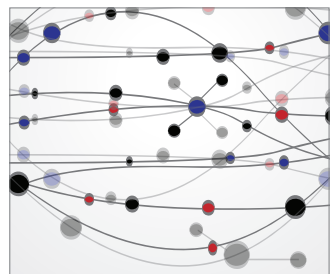

The Scientific World Journal
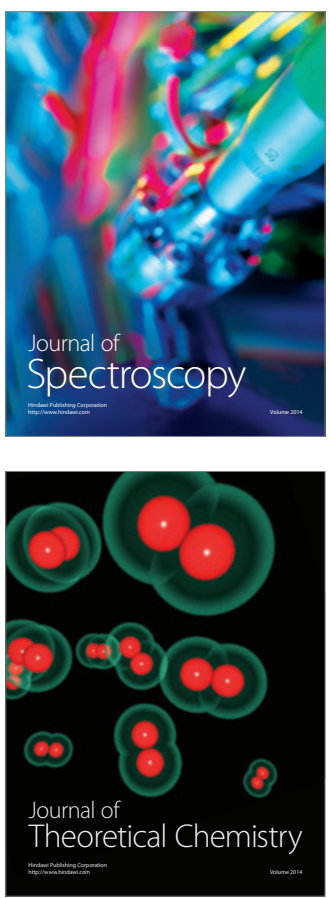
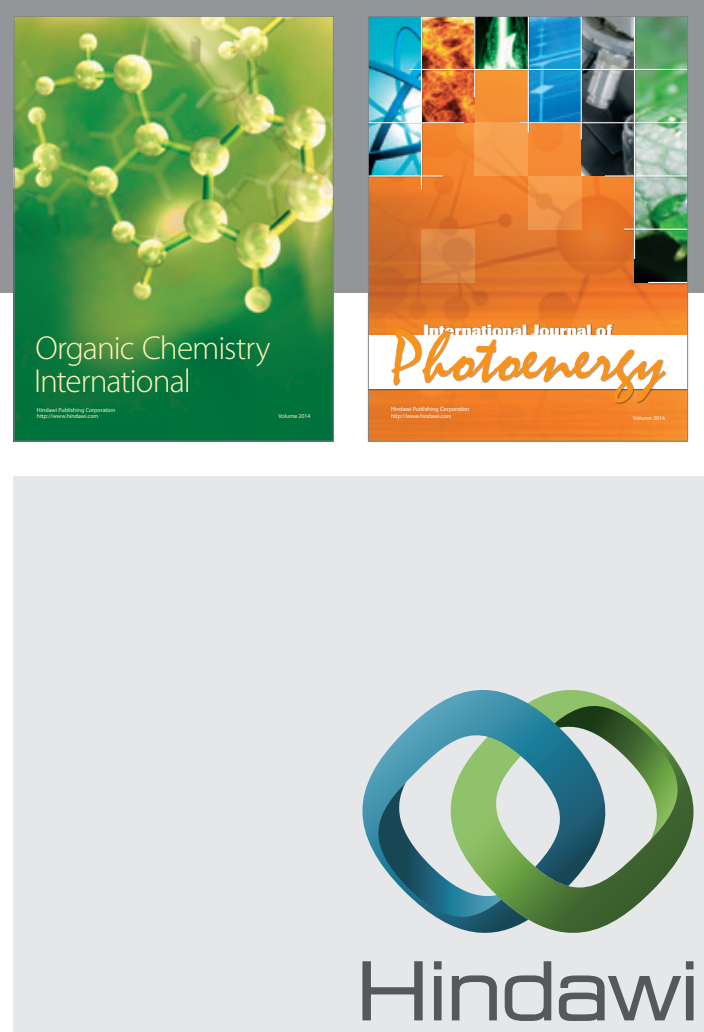

Submit your manuscripts at

http://www.hindawi.com

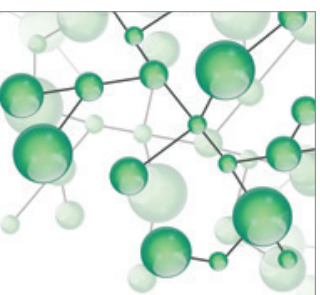

International Journal of

Inorganic Chemistry

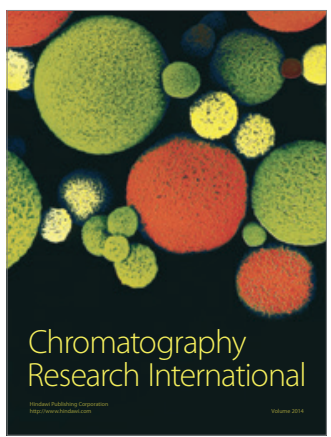

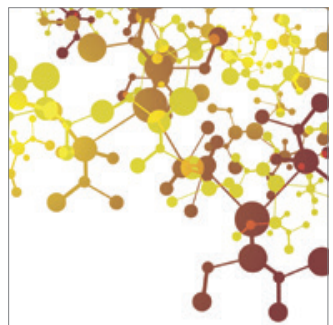

Applied Chemistry
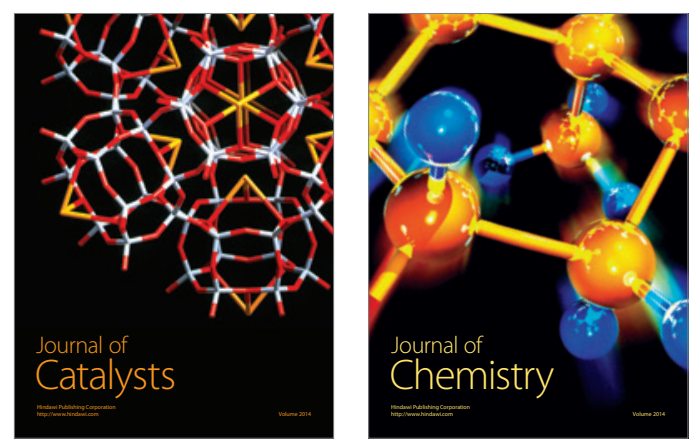
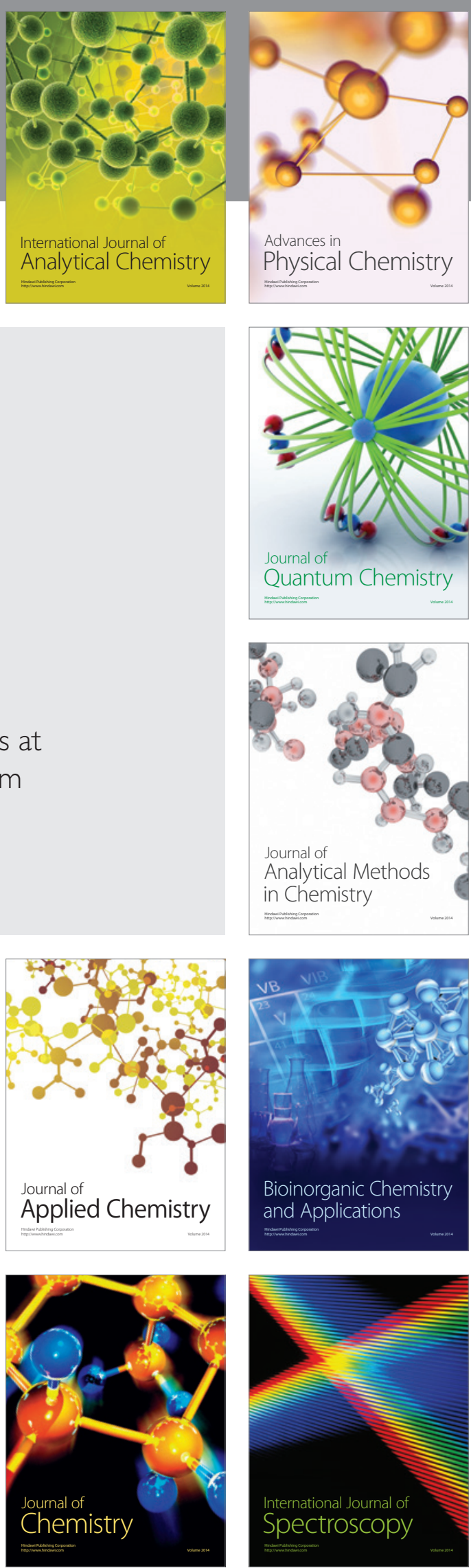